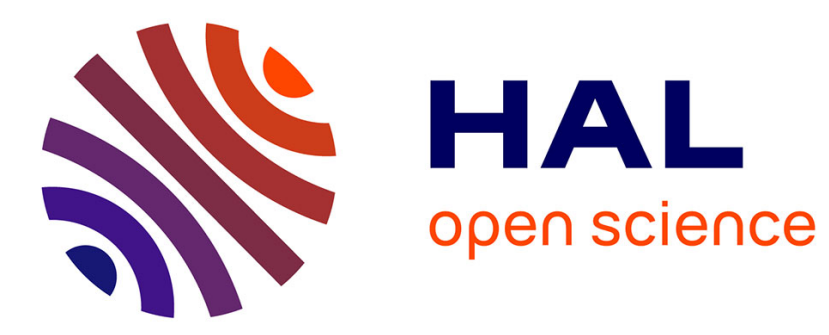

\title{
Phase-type aging modeling for health dependent costs
}

\author{
Maria Govorun, Guy Latouche, Stéphane Loisel
}

\section{To cite this version:}

Maria Govorun, Guy Latouche, Stéphane Loisel. Phase-type aging modeling for health dependent costs. Insurance: Mathematics and Economics, 2015, 62 (May 2015), pp.173-183. hal-01084274

\section{HAL Id: hal-01084274 \\ https://hal.science/hal-01084274}

Submitted on 18 Nov 2014

HAL is a multi-disciplinary open access archive for the deposit and dissemination of scientific research documents, whether they are published or not. The documents may come from teaching and research institutions in France or abroad, or from public or private research centers.
L'archive ouverte pluridisciplinaire HAL, est destinée au dépôt et à la diffusion de documents scientifiques de niveau recherche, publiés ou non, émanant des établissements d'enseignement et de recherche français ou étrangers, des laboratoires publics ou privés. 


\title{
Phase-type aging modeling for health dependent costs
}

\author{
Maria Govorun* Guy Latouche ${ }^{\dagger} \quad$ Stéphane Loisel ${ }^{\ddagger}$
}

November 5, 2014

\begin{abstract}
In the present paper we develop recursive algorithms to evaluate the distribution of the net present value (abbreviated as "NPV") of a health care contract. The duration of the program is a random variable representing the lifetime of an individual. We suggest a discrete time phase-type approach to model individual health care costs. In this approach, annual health care costs depend naturally on the health state of the individual. We also derive the distribution of the NPV assuming that annual health care costs are iid random variables. We demonstrate analytically that, under special parametrisation, the model with iid costs gives a similar expectation of the NPV to the one of the model with health dependent costs. We propose techniques to evaluate the impact of health related events and demonstrate it on numerical examples.

Keywords: Health dependent costs, net present value, phase-type aging process, Markov reward model, cost-effectiveness of a new treatment, cost of bad health
\end{abstract}

\section{Introduction}

Many life insurance companies are exposed to long term care risks. In opposition to classical health insurance where contracts are renewed every year

\footnotetext{
*Western University, Department of statistical and actuarial sciences, 262 Western Science Centre, 1151 Richmond Street, N6A 5B7 London, Ontario. Email: mgovorun@uwo.ca

${ }^{\dagger}$ Université Libre de Bruxelles, Département d'Informatique, CP 212, Boulevard du Triomphe, 1050 Bruxelles, Belgium. Email: latouche@ulb.ac.be

${ }^{\ddagger}$ Université Claude Bernard Lyon 1, Institut de Science Financière et dAssurances, 50 Avenue Tony Garnier, F-69007 Lyon, France. Email: stephane.loisel@univ-lyon1.fr
} 
and most lines of business are short-tailed, management of long term care contracts is complicated as it involves uncertainty about life and health of an individual over a long-term horizon. It is not only the uncertainty about the remaining life length which complicates the estimation of future health costs, but also the uncertainty related to the quality of this remaining lifetime, which is highly affected by the progress of medical science, by economical factors, etc.

Modelling health care costs is a problem of great interest in health insurance and health economics (Zhao and Zhou [2012]). The estimation of the costs plays a key role in pricing, reserving and risk assessment in health insurance, as well as performing cost-effectiveness and cost-utility analyses in health economics.

It is quite common to use multi-state Markov models for the problems arising in long term care. For example, in Guibert and Planchet [2014] the authors use a Markovian approach to construct inception rates with competing risks in long term care insurance where multiple pathologies are likely to cause disability. Also, Markovian models are widely used for the estimation of health care costs over a fixed period of time, where the changes of individual health states are taken into account. Specifically, it is assumed that health care costs depend on an individual health state, which is modelled by a Markov chain. In Castelli et al. [2007], Gardiner et al. [2006] and Zhao and Zhou [2012] the Markov chain has a fixed number of states, which is a subjectively chosen parameter, and does not depend on the age of an individual. The health care cost related quantities that are studied in Castelli et al. [2007], Gardiner et al. [2006] and Zhao and Zhou [2012] slightly differ from each other, but all are computed as expected values. In particular, Gardiner et al. [2006] work in continuous time and determine the expected net present value (abbreviated as "NPV") of health care costs over a fixed time horizon. In the present paper, we are focused on the distribution of NPV.

We develop models for NPV with our underlying assumption being that the lifetime and health of an individual are described by the phase-type distribution. Our motivation to use a phase-type representation for the lifetime of an individual came from Lin and Liu [2007]. Specifically, the authors define a finite-state continuous-time Markov process to represent the hypothetical aging process of an individual, this is called a phase-type aging model (below, "PH-aging model"). Aging is described as a process of consecutive transitions from one health state to another until death. One important property of this model is that the states have some physical interpretation, and their number is not chosen arbitrarily, it depends on mortality data through a well specified algorithmic procedure. Another important characteristic of the model, which 
makes it different from other phase-type models for health, and very relevant for actuarial applications at the same time, is that it provides a connection between the health state of an individual and his/her age. In our opinion, this property opens new horizons in health insurance and health economics. The model has been employed by different research groups: Su and Sherris [2012] use the model to study the impact of mortality heterogeneity on the insurance market, Govorun and Latouche [2014] and Govorun et al. [2011] use the model to estimate the financial impact of health on profits and losses of a pension fund.

We construct two discrete-time models designed to determine the distribution of NPV over a random phase-type horizon which is the lifetime of an individual. In our Markov reward model model, similarly to Castelli et al. [2007], Gardiner et al. [2006] and Zhao and Zhou [2012], we naturally assume that the annual health care costs depend on the health of an individual and that the value of the cost is a random variable or constant for each given health state. However, in reality not all types of health care costs are aging dependent. There is almost no age dependence for dental care costs as well as for costs related to a health damage caused by accidents. This is why in our second model we assume that the annual health care costs are independent and identically distributed random variables. In insurance, this approach might be useful to estimate personal accident type of policies. The model with iid costs is also useful when there is not enough data to evaluate the Markov reward model.

In the two models, NPV is a sum of discounted random health costs, where the number of terms in the sum has a phase-type distribution. In the absence of discounting and if the health costs are iid, in order to find the distribution of NPV one may apply the algorithm suggested by Eisele [2006], which is a natural extension of the widely used procedure introduced by Panjer [1981]. In our work, we develop a method that enables us to write similar algorithms for the distribution of NPV in both models. We suggest a parametrization procedure, and we show that, under a special choice of parameters, the two models give approximately same results for the NPV.

We provide numerical illustrations to show the flexibility of the Markov reward model for costs. In our first example, we use the model to estimate the financial impact of longer survival in bad health states. In the second example we demonstrate the applicability of the model in health economics by estimating cost-effectiveness of different treatment strategies (see Drummond et al. [2005]). In the third example, we study the impact of market fluctuations by performing sensitivity analysis with respect to discounting.

The paper is structured as follows. In Section 2, we describe the phasetype assumption for the lifetime by presenting the model of Lin and Liu 


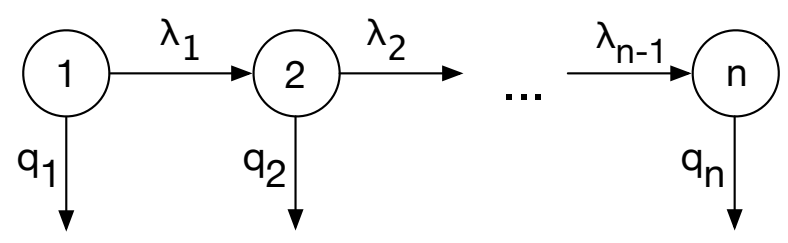

Figure 1: Phase-type aging process

[2007]. In Section 3, we introduce our model with random health dependent costs and consider a special case when cost is a constant for a given state. We also present the model with iid costs, and in Section 4 we suggest a parametrization procedure for both models for costs that results in a similar expected NPV. In Section 5, we demonstrate the flexibility of the phase-type approach on numerical examples. We present our conclusions and future research directions in Section 6.

\section{Phase-type lifetime}

Lin and Liu [2007] define a finite-state continuous-time Markov process to model the hypothetical aging process of an individual. Aging is described as a process of consecutive transitions from one health state to another until death, as shown on Figure 1. There, the system has $n$ phases with the transition rates $\lambda_{i}$ from $i$ to $i+1$, for $i=1, \ldots, n-1$, and the transition rates $q_{i}$ to the absorbing phase, which is interpreted as the state of death of the individual: the time to reach the absorbing phase is interpreted as the lifetime of the individual.

The generator is

$$
Q=\left[\begin{array}{cc}
\Lambda & q \\
\underline{0}^{T} & 0
\end{array}\right]
$$

where $\underline{q}=\left[\begin{array}{llll}q_{1} & q_{2} & \ldots & q_{n}\end{array}\right]^{\mathrm{T}}$ and

$$
\Lambda=\left[\begin{array}{ccccc}
-\left(\lambda_{1}+q_{1}\right) & \lambda_{1} & 0 & \ldots & 0 \\
0 & -\left(\lambda_{2}+q_{2}\right) & \lambda_{2} & \ldots & 0 \\
0 & 0 & -\left(\lambda_{3}+q_{3}\right) & \ddots & 0 \\
\vdots & \vdots & \ddots & \ddots & \vdots \\
0 & 0 & 0 & \ldots & -q_{n}
\end{array}\right]
$$

Newborns start from state 1 , thus the initial probability vector is $\left[\underline{\alpha}^{\mathrm{T}}, 0\right]$ with $\underline{\alpha}=\left[\begin{array}{llll}1 & 0 & \ldots & 0\end{array}\right]^{\mathrm{T}}$. The transition rates have a special structure, it 
suffices here to mention that they accommodate a developmental period for very young ages and a period of higher accident probability in the mid-range of childhood. Some of the rates are constant, thus although the model potentially consists of a large number of parameters, 9 to 13 different parameters are often enough to give a good approximation of mortality data. For a more detailed explanation of the parameters structure and fitting, we refer the reader to Lin and Liu [2007] and Govorun and Latouche [2014].

The distribution of the Markov process at time $t$ is given by $\underline{\alpha}^{\mathrm{T}} e^{Q t}$, which is equal to $\left[\underline{\alpha}^{\mathrm{T}} e^{\Lambda t}, 1-\underline{\alpha}^{\mathrm{T}} e^{\Lambda t} \underline{1}\right]$, this means the following: $\left(\underline{\alpha}^{\mathrm{T}} e^{\Lambda t}\right)_{i}$ is the probability to survive for $t$ years and to be in the phase $i$ at time $t, 1-\underline{\alpha}^{\mathrm{T}} e^{\Lambda t} \underline{1}$ is the probability to have died before time $t$.

One important feature of the model, which makes it different from other phase-type models for health, is that it provides a connection between the age of an individual and his/her health states. Specifically, if we denote the health distribution at age $x$ as $\underline{\tau}_{x}$, we obtain from the model that

$$
\underline{\tau}_{x}^{\mathrm{T}}=\left(\underline{\alpha}^{\mathrm{T}} e^{\Lambda x} \underline{1}\right)^{-1} \underline{\alpha}^{\mathrm{T}} e^{\Lambda x}
$$

Therefore, the time until death of an individual of age $x$ follows the phasetype distribution with parameters $\left(\underline{\tau}_{x}, \Lambda\right.$ ) (see Latouche and Ramaswami [1999]) and the phase distribution of the individual after $t$ units of time is $\tau_{x}^{\mathrm{T}} e^{\Lambda t}$. The probability that such an individual survives for $t$ units of time is given by

$$
S_{x}(t)=\underline{\tau}_{x}^{\mathrm{T}} e^{\Lambda t} \underline{1} .
$$

Denote by $\bar{L}^{(i)}$ the expected remaining life for an individual in physiological state $i$. It is given by

$$
\bar{L}^{(i)}=\underline{\alpha}^{(i)}\left(I-e^{\Lambda}\right)^{-1} \underline{1},
$$

where $\underline{\alpha}^{(i)}$ is a row-vector of size $n$ with

$$
\alpha_{i}^{(i)}=1, \quad \alpha_{j}^{(i)}=0 \text { for } j \neq i .
$$

The expected remaining lifetime for an individual aged $x$ is

$$
\bar{L}_{x}=\underline{\tau}_{x}^{\mathrm{T}}\left(I-e^{\Lambda}\right)^{-1} \underline{1} .
$$

\section{Markov Reward Model for health costs}

Our main objective is to obtain the distribution of the NPV of a health care contract given by

$$
S=\sum_{t=1}^{[L]} v^{t-1} X_{t}
$$


where $[L]$ is the integer number of remaining years of life, and $X_{t}$ is the health care cost in year $t$. The coefficient $v$ is allowed to take any positive value including greater than one, so as to include inflation, interest force, the increase of health care prices, etc.

Denote by $\psi_{t}$ the health state of the individual at time $t$. The distribution of $\psi_{t}$ is given by

$$
\mathrm{P}\left[\psi_{t}=i\right]=\left(\underline{\tau}^{\mathrm{T}} e^{\Lambda t}\right)_{i}, \quad i=1, \ldots, n .
$$

Here, $\Lambda$ is the generator of a phase-type aging process of a two-diagonal (see $(2))$ or any other structure, $(\underline{\tau})_{i}=\mathrm{P}\left[\psi_{0}=i\right]$ is the health state distribution of the individual at time 0. From Eq. (3), $\underline{\tau}=\underline{\tau}_{x}$ for an individual aged $x$.

We focus on the distribution of $S$, which we denote by $H_{S}(k), k \geq 0$. The problem of determining $H_{S}(k)$ is equivalent to the problem of finding the conditional distribution given the health state at time 0 , because $H_{S}(k)=$ $\underline{\tau}^{\mathrm{T}} \underline{H}(k)$, where

$$
\underline{H}(k): H_{i}(k)=\mathrm{P}\left[S \leq k \mid \psi_{0}=i\right], \quad i=1, \ldots, n .
$$

Denote by $y$ the conditional probability to die in the first year, given the health state at time 0 . It is complement to the one year survival probability, so that

$$
\underline{y}=\underline{1}-e^{\Lambda} \underline{1} .
$$

For annual costs $X_{t}$ we define a Markov reward model (abbreviated as "MRM"). It is convenient to introduce the model as a triplet $(A \cup\{D\}, \underline{\mathcal{W}}, P)$, where

1. $A \cup\{D\}$ is the set of possible states: $A$ is the set of $n$ health states from the aging model, $\{D\}$ is one absorbing state for death;

2. $\underline{\mathcal{W}}$ is the set of $n+1$ variables representing annual cost in different health states. We assume that, for $i \in A, \mathcal{W}_{i}, i=1, \ldots, n$ is a discrete random variable: it is defined on a given set $\mathcal{C}=\left\{c_{1}, \ldots, c_{M}\right\}$ of non-negative values with a distribution which may depend on $i$. For $i \in\{D\}, \mathcal{W}_{n+1}$ is zero with probability one. We denote by $F^{(i)}(k)$ and by $f^{(i)}(k)$ the cumulative distribution function and the probability mass function of $\mathcal{W}_{i}$, respectively.

3. $P$ is the one year transition probability matrix for the states, which is constructed as follows

$$
P=\left[\begin{array}{ll}
e^{\Lambda} & y \\
\underline{0}^{\mathrm{T}} & 1
\end{array}\right],
$$

where $\underline{y}$ is given by Eq. (9). 
Theorem 3.1 Suppose that $\left\{X_{t}\right\}$ follows the Markov Reward model defined by the triplet $(A \cup\{D\}, \underline{\mathcal{W}}, P)$. The conditional distribution $\underline{H}(k)$ of the net present value $S$, defined by Eq. (7), is

$$
H_{i}(k)=y_{i} F^{(i)}(k)+\left(e^{\Lambda}\right)_{(i, \cdot)} \sum_{j=1}^{M} f^{(i)}\left(c_{j}\right) \underline{H}\left(\frac{k-c_{j}}{v}\right), \quad k \geq 0, \quad i=1, . ., n,
$$

where $\underline{y}$ is given by Eq. (9).

Proof. We apply a renewal argument to obtain the recursive expression for $\underline{H}(k)$. We notice that

$$
\begin{aligned}
& \text { if } \quad[L]=1 \quad \text { then } \quad S=X_{1}, \\
& \text { if } \quad[L] \geq 2 \text { then } \quad S=X_{1}+v \tilde{S},
\end{aligned}
$$

where $\tilde{S}$ is a random variable with the same transition matrix as $S$, but with a different initial health state vector. Thus, we have

$$
\begin{aligned}
H_{i}(k)= & \mathrm{P}\left[[L]=1 \mid \phi_{0}=i\right] \mathrm{P}\left[X_{1} \leq k \mid \phi_{0}=i\right]+ \\
& \sum_{s \in\{A\}} \mathrm{P}\left[\phi_{1}=s \mid \phi_{0}=i\right] \mathrm{P}\left[X_{1}+v \tilde{S} \leq k \mid \phi_{1}=s, \phi_{0}=i\right] \\
= & y_{i} F^{(i)}(k)+\sum_{s \in\{A\}}\left(e^{\Lambda}\right)_{(i, s)} \sum_{j=1}^{M} f^{(i)}\left(c_{j}\right) H_{s}\left(\frac{k-c_{j}}{v}\right) \\
= & y_{i} F^{(i)}(k)+\left(e^{\Lambda}\right)_{(i, \cdot)} \sum_{j=1}^{M} f^{(i)}\left(c_{j}\right) \underline{H}\left(\frac{k-c_{j}}{v}\right) .
\end{aligned}
$$

In the theorem below we determine a closed form expression for the expectation of $S$. Denote by $\rho(\cdot)$ the spectral radius operator.

Theorem 3.2 If $v \neq \rho^{-1}\left(e^{\Lambda}\right)$, the expectation of $S$ in the Markov reward model $(A \cup\{D\}, \underline{\mathcal{W}}, P)$ is

$$
E[S]=\underline{\tau}^{T}\left(I-v e^{\Lambda}\right)^{-1} E\left[\underline{\mathcal{W}}_{A}^{T}\right]
$$

where $\underline{\tau}$ is the initial health state distribution, $\Lambda$ is the generator of the corresponding phase-type aging process.

Proof. Denote by $g(\zeta)=E\left[\zeta^{S}\right]$ the probability generation function of $S$. We have

$$
g(\zeta)=\underline{\tau}^{\mathrm{T}} \underline{\sigma}(\zeta)
$$


where $\underline{\sigma}(\zeta)$ is a column vector of size $n$ with $\sigma_{i}(\zeta)=E\left[\zeta^{S} \mid \phi_{0}=i\right], i \in A$ and thus

$$
E[S]=\left.\frac{\partial g(\zeta)}{\partial \zeta}\right|_{\zeta=1}=\left.\underline{\tau}^{\mathrm{T}} \frac{\partial \underline{\sigma}(\zeta)}{\partial \zeta}\right|_{\zeta=1} .
$$

We have

$$
\begin{aligned}
\sigma_{i}(\zeta)= & \sum_{j \in A \cup\{D\}} \mathrm{P}\left[\phi_{1}=j \mid \phi_{0}=i\right] E\left[\zeta^{S} \mid \phi_{0}=i, \phi_{1}=j\right] \\
= & \sum_{j \in A} \mathrm{P}\left[\phi_{1}=j \mid \phi_{0}=i\right] E\left[\zeta^{X_{1}+v \tilde{S}} \mid \phi_{0}=i, \phi_{1}=j\right] \\
& +\sum_{j \in\{D\}} \mathrm{P}\left[\phi_{1}=j \mid \phi_{0}=i\right] E\left[\zeta^{X_{1}} \mid \phi_{0}=i, \phi_{1}=j\right] \\
= & f_{i}(\zeta) \sum_{j \in A} \mathrm{P}\left[\phi_{1}=j \mid \phi_{0}=i\right] E\left[\left(\zeta^{v}\right)^{\tilde{S}} \mid \phi_{1}=j\right]+f_{i}(\zeta) y_{i} \\
= & f_{i}(\zeta) \sum_{j \in A}\left(e^{\Lambda}\right)_{(i, j)} \sigma_{j}\left(\zeta^{v}\right)+f_{i}(\zeta) y_{i}
\end{aligned}
$$

where $f_{i}(\zeta)=E\left[\zeta^{\mathcal{W}_{i}}\right]$. In matrix form, we write

$$
\underline{\sigma}(\zeta)=\Upsilon(\zeta) e^{\Lambda} \underline{\sigma}\left(\zeta^{v}\right)+\Upsilon(\zeta) \underline{y}
$$

with

$$
\Upsilon(\zeta)=\left[\begin{array}{ccc}
f_{1}(\zeta) & \ldots & 0 \\
\vdots & \ddots & \vdots \\
0 & \ldots & f_{n}(\zeta)
\end{array}\right]
$$

Therefore,

$$
\frac{\partial \underline{\sigma}(\zeta)}{\partial \zeta}=\Upsilon^{\prime}(\zeta) e^{\Lambda} \underline{\sigma}\left(\zeta^{v}\right)+\left.v \zeta^{v-1} \Upsilon(\zeta) e^{\Lambda} \frac{\partial \underline{\sigma}(z)}{\partial z}\right|_{z=\zeta^{v}}+\Upsilon^{\prime}(\zeta) \underline{y}
$$

which we evaluate at $\zeta=1$ to obtain, using $\underline{\sigma}(1)=\underline{1}$,

$$
\left.\frac{\partial \underline{\sigma}(\zeta)}{\partial \zeta}\right|_{\zeta=1}=\Upsilon^{\prime}(1)\left(e^{\Lambda} \underline{1}+\underline{y}\right)+\left.v e^{\Lambda} \frac{\partial \underline{\sigma}(z)}{\partial z}\right|_{z=1},
$$

with

$$
\Upsilon^{\prime}(1)=\left[\begin{array}{ccc}
E\left[\mathcal{W}_{1}\right] & \ldots & 0 \\
\vdots & \ddots & \vdots \\
0 & \ldots & E\left[\mathcal{W}_{n}\right]
\end{array}\right]
$$


After rearranging the terms, using Eq. (9), we obtain

$$
\left.\frac{\partial \underline{\sigma}(\zeta)}{\partial \zeta}\right|_{\zeta=1}=\left(I-v e^{\Lambda}\right)^{-1} E\left[\underline{\mathcal{W}}_{A}^{\mathrm{T}}\right]
$$

Clearly, the inverse of $\left(I-v e^{\Lambda}\right)$ exists when $v \neq \rho^{-1}\left(e^{\Lambda}\right)$. By combining Eq. (14) and Eq. (19) we obtain the statement of the lemma.

A particular case of the MRM is the deterministic Markov reward model $(A \cup\{D\}, \underline{W}, P)$, where $\underline{W}$ is the vector of size $n+1$ of constant health care costs for each state so that

$$
W_{i} \geq 0 \text {, if } i \in A \text {, and } W_{i}=0 \text {, if } i \in\{D\} .
$$

The recursive formula for $S$ in this case is given by the corollary below and results directly from Theorem 3.1.

Corollary 3.3 Suppose that $X_{t}$ follows the Markov Reward Model ( $A \cup$ $\{D\}, \underline{W}, P)$ with $\underline{W}$ defined by (20). The conditional distribution $\underline{H}(k)$ of the net present value $S$ of a health care contract defined by Eq. (7) is such that

$$
H_{i}(k)=y_{i} \mathbb{1}_{\left\{W_{i} \leq k\right\}}+\left(e^{\Lambda}\right)_{(i, \cdot)} \underline{H}\left(\frac{k-W_{i}}{v}\right), \quad k \geq 0,
$$

where $\underline{y}$ is given by Eq. (9), $i=1, . ., n$. In particular,

$$
\begin{array}{ll}
\text { if } W_{i} \neq 0 \quad \text { then } & H_{i}(0)=0, \\
\text { if } W_{i}=0 \quad \text { then } & H_{i}(0)=y_{i}+\left(e^{\Lambda}\right)_{(i, \cdot)} \underline{H}(0) .
\end{array}
$$

The closed form expression for the expectation of $S$ is given in the corollary below and results directly from Theorem 3.2.

Corollary 3.4 If $v \neq \rho^{-1}\left(e^{\Lambda}\right)$, the expectation of $S$ for the Markov Reward Model $(A \cup\{D\}, \underline{W}, P)$ with $\underline{W}$ defined by (20) is

$$
E[S]=\underline{\tau}^{T}\left(I-v e^{\Lambda}\right)^{-1} \underline{W}_{A}^{T},
$$

where $\underline{\tau}$ is the initial health state distribution, $\Lambda$ is the generator of the corresponding phase-type aging process and $\rho(\cdot)$ is a spectral radius operator.

\section{Model with iid costs}

For some types of health care costs it is convenient to assume that annual health care costs $X_{t}$ are random, independent and have the same distribution. We denote by $X$ a random variable with this common distribution. This assumption leads to the following theorems. 
Theorem 3.5 (iid costs) Suppose that $X_{t}$ are discrete iid random variables distributed as $X$ that takes $M$ non-negative possible values, $X \in\left\{c_{1}, \ldots, c_{M}\right\}$, with $F(k)=P[X \leq k]$ and $f(k)=P[X=k]$. The conditional distribution $\underline{H}(k)$ of the net present value $S$ of a health care contract defined by Eq. (7) is such that

$$
H_{i}(k)=y_{i} F(k)+\left(e^{\Lambda}\right)_{(i, \cdot)} \sum_{\theta=1}^{M} f\left(c_{\theta}\right) \underline{H}\left(\frac{k-c_{\theta}}{v}\right), \quad k \geq 0,
$$

where $\underline{y}$ is given by Eq. (9), $i=1, \ldots, n$. In particular,

$$
H_{i}(0)=f(0) \underline{\alpha}^{(i)} e^{\Lambda}\left(I-f(0) e^{\Lambda}\right)^{-1} \underline{y},
$$

where $\underline{\alpha}^{(i)}$ is given by Eq. (5).

Proof. The proof is based on the representation of $S$ given by (11) and (12) and analogous to the proof of Theorem 3.1 with $F^{(i)}(k)=F(k)$ and $f^{(i)}(k)=$ $f(k)$ for $i=1, \ldots, n$. If $k=0$, this implies that all costs $X_{t}, t=1, \ldots,[L]$ are equal to zero and, therefore,

$$
\begin{aligned}
H_{i}(0) & =\sum_{s=1}^{\infty} \mathrm{P}[[L]=s](f(0))^{s}=\sum_{s=1}^{\infty} \underline{\alpha}^{(i)} e^{\Lambda s} \underline{y}(f(0))^{s} \\
& =\underline{\alpha}^{(i)} f(0) e^{\Lambda} \sum_{s=0}^{\infty} e^{\Lambda s} \underline{y}(f(0))^{s} \\
& =f(0) \underline{\alpha}^{(i)} e^{\Lambda}\left(I-f(0) e^{\Lambda}\right)^{-1} \underline{y} .
\end{aligned}
$$

We note that the existence of the inverse of $\left(I-f(0) e^{\Lambda}\right)$ is guaranteed by $f(0)$ being at most one and $\rho\left(e^{\Lambda}\right)$ being less than one.

Theorem 3.6 If $v \neq \rho^{-1}\left(e^{\Lambda}\right)$, the expectation of $S$ in the model with iid costs $X$ is

$$
E[S]=\underline{\tau}^{T}\left(I-v e^{\Lambda}\right)^{-1} \underline{1} E[X] .
$$

Proof. If $v=1$, we use the independency between $X$ and $L$, and Eq. (6) to obtain

$$
E[S]=E\left[\sum_{t=1}^{[L]} X_{t}\right]=E[X] E[L]=\underline{\tau}^{\mathrm{T}}\left(I-e^{\Lambda}\right)^{-1} \underline{1} E[X] .
$$

If $v \neq 1$, the expectation of $S$ is computed as follows

$$
E[S]=E\left[\sum_{t=1}^{[L]} v^{t-1} X_{t}\right]=E[X] E\left[\frac{1-v^{[L]}}{1-v}\right]=\frac{1}{1-v}(1-g(v)) E[X]
$$


where $g(v)$ is the generating function of a discrete phase-type random variable given, for example, in Latouche and Ramaswami [1999] as

$$
g(v)=v \underline{\tau}^{\mathrm{T}}\left(I-v e^{\Lambda}\right)^{-1}\left(\underline{1}-e^{\Lambda} \underline{1}\right) .
$$

One can represent 1 as $\underline{\tau}^{\mathrm{T}}\left(I-v e^{\Lambda}\right)^{-1}\left(I-v e^{\Lambda}\right) \underline{1}$. Therefore,

$$
\begin{aligned}
1-g(v) & =\underline{\tau}^{\mathrm{T}}\left(I-v e^{\Lambda}\right)^{-1}\left(I-v e^{\Lambda}\right) \underline{1}-v \underline{\tau}^{\mathrm{T}}\left(I-v e^{\Lambda}\right)^{-1}\left(\underline{1}-e^{\Lambda} \underline{1}\right) \\
& =\underline{\tau}^{\mathrm{T}}\left(I-v e^{\Lambda}\right)^{-1}\left(I-v e^{\Lambda}-v I+v e^{\Lambda}\right) \underline{1} \\
& =(1-v) \underline{\tau}^{\mathrm{T}}\left(I-v e^{\Lambda}\right)^{-1} \underline{1} .
\end{aligned}
$$

This result together with Eq. (25) gives us the statement of the lemma.

\section{Approximation and parametrization}

In this section, we discuss interesting parametrization aspects of the Markov Reward Model and the model with iid costs. Often, in practice there is not enough data to estimate the Markov Reward Model $(A \cup\{D\}, \underline{\mathcal{W}}, P)$, so we focus on the parametrization of the MRM $(A \cup\{D\}, \underline{W}, P)$ with constant costs for a given state. The discussion about the parametrization of the general MRM we refer to Govorun et al. [2014].

Denote by $\hat{Y}_{x}$ the health care cost for age $x$, observed from the data, and denote by $M$ an integer such that $\hat{Y}_{x} \leq M$ (with probability one) for all $x$. In order to fully parametrize the Markov Reward Model, one needs to determine a value of the cost $W_{i}$ for every health state $i \in A$. One method to determine these values is to match the expectations of the costs for all ages. For example, one can solve numerically the linear optimization problem

$$
\min _{\underline{W}_{A}}\left\|\mathcal{T} \underline{W}_{A}-\mathcal{J}\right\|_{2}^{2}
$$

where $\mathcal{T}=\left[\underline{\tau}_{x}^{\mathrm{T}}\right]_{x=1, . ., \infty}$ and $\mathcal{J}=\left[E\left[\hat{Y}_{x}\right]\right]_{x=1, . ., \infty}$. We note that, as shown in Lin and Liu [2007], the number of states in $A$ should be around 200 for a good approximation of mortality rates. The number of ages in data can not exceed, in general, 120. This is why the unconstrained optimisation (26) leads to numerous and physically unlikely situations. Thus, we have found it useful to impose the following natural constraint $\underline{W}_{A}: 0<W_{i} \leq$ $W_{i+1} \leq M, i=1, \ldots, n-1$. We find it important to underline here that if we consider health related expenditures $\hat{Y}_{x}$ that have a non decreasing trend by age, this property together with the ordering constraint provides one with a good looking solution to (26). 
For the Model with iid costs one needs to determine a common distribution for the costs $X$ of successive years. An individual aged $x$ in the beginning of a health care contract will be alive for unknown number of years, which signifies that $X$ might be considered as a combination of health care costs for future years of life:

$$
X=\sum_{j=x}^{\infty} \beta_{j-x} \hat{Y}_{j}
$$

The weights $\beta_{j}$ can be chosen in many different ways that we discuss later in the section. In the proposition below, we show that there exists a special choice of $\beta_{j}$ in the Model with iid costs that gives approximately same expectation of $S$ as in the Markov Reward Model.

Proposition 4.1 Denote by $S$ the NPV in the MRM model with (26) and by $S^{(\beta)}$ the NPV of the health care costs in the model with iid costs with parametrization (27). If the weights $\beta_{j}, j=0, . ., \infty$ are chosen such that

$$
\beta_{j}=\frac{v^{j} S_{x}(j)}{\sum_{j \geq 0} v^{j} S_{x}(j)}
$$

then

$$
E[S]-E\left[S^{(\beta)}\right]=\sum_{j=0}^{\infty} v^{j} S_{x}(j)\left(\left(\mathcal{T} \underline{W}_{A}\right)_{x+j}-E\left[\hat{Y}_{x+j}\right]\right) .
$$

The difference between the expectations only depends on the quality of the fitting (26).

Proof. We first write the expectation of $S$ in the MRM as

$$
\begin{aligned}
E[S]=\sum_{i \geq 0} v^{i} \underline{\tau}_{x}^{\mathrm{T}} e^{\Lambda i} \underline{W}_{A} & =\sum_{i \geq 0} v^{i} \underline{\tau}_{x}^{\mathrm{T}} e^{\Lambda i} \underline{1}_{x+i}^{\mathrm{T}} \underline{W}_{A} \\
& =\sum_{i \geq 0} v^{i} S_{x}(i)\left(\mathcal{T} \underline{W}_{A}\right)_{x+i}
\end{aligned}
$$

where $\left(\mathcal{T} \underline{W}_{A}\right)_{j}$ is the average health cost at age $j$ from Eq. (26). Secondly, we derive the expectation of $S$ in the Model with iid costs and weights $\beta_{j}$ 
chosen as in Eq. (28)

$$
\begin{aligned}
E\left[S^{(\beta)}\right] & =E\left[X^{(\beta)}\right] \sum_{i \geq 0} v^{i} S_{x}(i) \\
& =\left(\sum_{j \geq 0}^{\infty} \beta_{j} E\left[\hat{Y}_{j+x}\right]\right)\left(\sum_{i \geq 0} v^{i} S_{x}(i)\right) \\
& =\sum_{j \geq 0}^{\infty} v^{j} S_{x}(j) E\left[\hat{Y}_{x+j}\right] .
\end{aligned}
$$

Clearly, in Eq. (31) and Eq. (33) the terms $\left(\mathcal{T} \underline{W}_{A}\right)_{x+j}$ and $E\left[\hat{Y}_{x+j}\right]$ are only approximately the same as a result from the minimization procedure (26).

We give to $\beta_{j}$ the interpretation of "the importance of each year". The weights (28) suggest that the importance of future years depends on the survival probabilities and on economical factors. If $v<1$, future years have less importance; if $v>1$, they get more weight. Depending on particular needs and purposes, $\beta_{j}$ can be chosen uniform or independent of discounting (by setting $v=1$ in (28)).

\section{Cost of health. Numerical illustrations.}

There are many health and non-health related events that may lead to significant changes in future health development and, therefore, affect future profits and losses of a health care contract. In this section, we present model examples to demonstrate potential and sensitivity of the proposed Markov reward model for costs. We provide our examples for the deterministic Markov reward model (20), as one can naturally extend them to the general Markov reward model case.

In our examples, we assume that health care costs $\hat{Y}_{x}$ are available for each age, from 0 to 100, as averages only. We take $M$ from Section 4 to be equal to 10 and assume that $\hat{Y}_{x}$ has $E\left[\hat{Y}_{0}\right]=1, E\left[\hat{Y}_{100}\right]=10$ and $E\left[\hat{Y}_{x}\right]$ is linear in between. As our mortality model, we take the phase-type aging model of Section 2 with parameters estimated in Lin and Liu [2007] for 1911 Swedish cohort of males with the number of states $n=200$, and we consider an individual aged 40 at time zero so that his initial health state distribution $\underline{\tau}_{40}$ is given by (3) with $x=40$.

We apply the fitting procedure (26) to the average costs $E\left[\hat{Y}_{x}\right]$ by age to determine the costs $\underline{W}_{A}$ by state, which we present in Fig. 2. As we are mostly interested in values of $\underline{W}_{A}$ that provide a good fit to $E\left[\hat{Y}_{x}\right]$, we do not give detailed comments about the obtained values of $\underline{W}_{A}$, we only remark 


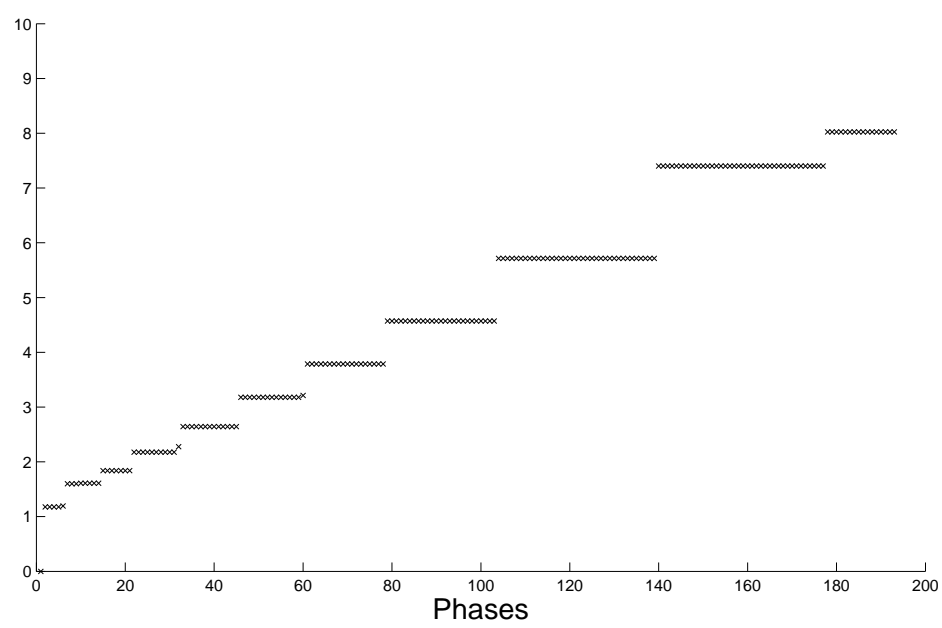

Figure 2: Health care costs $W_{i}$ for states $i \in\{A\}$ Markov reward model, $M=10$, SW1911M

that the piecewise constant step structure is guaranteed by the constraint $\underline{W}_{A}: 0<W_{i} \leq W_{i+1} \leq 10, i=1, \ldots, n-1$.

To give an impression of the fitting quality, in Fig. 3 we show $E\left[\hat{Y}_{x}\right]$ and compare it to $\left(\mathcal{T} \underline{W}_{A}\right)_{x}$ for $x=1, \ldots, 100$. One can observe that the fit of average costs is quite good, especially for young ages. The total sum of the squared differences between the average costs is 0.6242 with maximum cost value being 10 .

We compute the distribution of $S$ from Corollary 3.3 with the obtained values of $\underline{W}_{A}$, and we present the distribution as a solid line in both Fig. 4 and Fig. 5 for $v=1$ and $v=1.02$, respectively. Below, we construct two scenarios of future health development in order to examine the sensitivity of the distribution.

Cost of living with bad health. A phenomenon, which becomes more important with new developments in medicine and the accompanying change in mortality, is that people survive longer in older ages and in bad health states. To estimate the financial impact of such longevity, we suggest to decrease by $\varepsilon$ the mortality rates $q_{i}, i=1, \ldots, n$ of the matrix $\Lambda$ for the last $m$ states. In Fig. 4 we present the distributions of $S$, computed using Theorem 3.3, taking $v=1$ and $m=30$ and different values of $\varepsilon$. One observes from the figure that the decrease of mortality rates for the last 30 health states leads to the change of the distribution starting with cost 200 approximately. This indicates that the individuals who experience the change of mortality rates also spend more than 200. This amount may be compared to the 


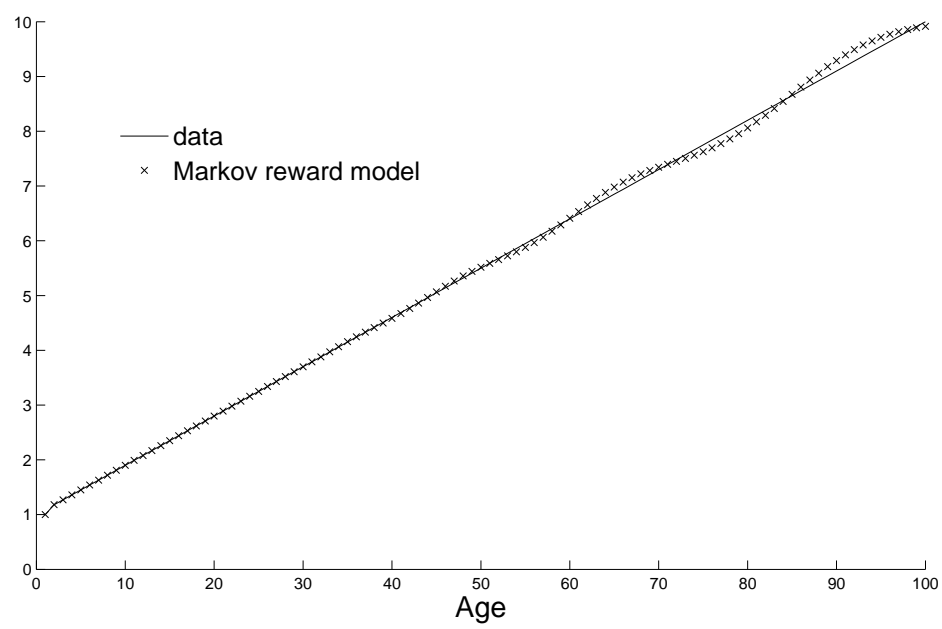

Figure 3: Expected health care costs for ages 1-100

$$
M=10, \text { SW1911M }
$$

expected cost until reaching state $n-m$. In our examples, the individuals have age 40 at the start of the contract, which implies that their expected health state is about 90 . The average time spent in state $i$ equals $1 /\left|\Lambda_{i i}\right|$ and the health cost in state $i$ is $W_{i}$. Thus, the expected cost accumulated from state 90 to state $n-m=170$ is given by $\sum_{i=90}^{170} W_{i} /\left|\Lambda_{i i}\right| \simeq 209$, which confirms the observation.

Cost-effectiveness of a new treatment. Another problem that can be analysed by the suggested approach and that might be of interest to health economists, is the estimation of the financial impact of a new costly treatment strategy. Let costs $\underline{W}_{A}$ for health states represent average health care costs of a general type, i.e. we assume that they include the costs of drugs, treatments and all medical examinations. The invention of a new expensive drug or an X-ray machine, for example, can increase the costs significantly, and does not necessarily lead to a significant increase of life length. Let us consider an example.

The average health state for $\underline{\tau}_{40}$ is around 90 out of 200 , the average life expectancy $\bar{L}_{40}$ given by (6) is 37.5 , and the expected value of $S$ is 413 . We show the cumulative distribution function of $S$ as a solid line in Fig. 5.

We compute $\bar{L}_{40}, E[S]$ and the distribution of $S$ for increased costs $W_{i}$ for states 1 to $k$ by $c$ units and by decreasing the aging rates $\lambda_{i}$ s in (2) by $\delta$ percent for states $k+1$ to $n$. For $c=5$, the results are 


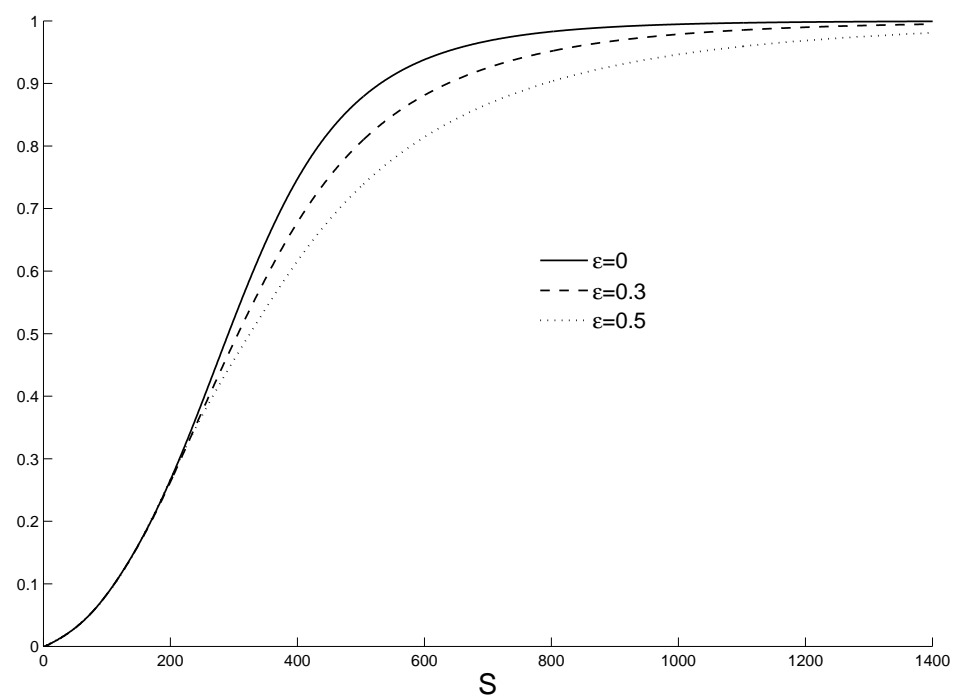

Figure 4: Markov reward model: distribution of $S$ with mortality rates decreased for the last 30 states by $\varepsilon$ $v=1, W_{i}, i \in\{A\}$ from Fig. 2, SW1911M

\begin{tabular}{l|c|c} 
& $\bar{L}_{40}$ & $E[S]$ \\
\hline no change & 37.5 & 413 \\
\hline$\delta=10 \%, k=100$ & 39.8 & 478 \\
\hline$\delta=50 \%, k=150$ & 41.5 & 656
\end{tabular}

The table shows that the average price one needs to pay for extending the lifetime by 2.3 years is 65 units or $15 \%$ of NPV, by 4 years - 243 units or $59 \%$ of NPV. The corresponding distributions of $S$ are shown in Fig. 5, as a dashed and a dotted line, respectively. Clearly, the strategy with $\delta=50 \%, k=150$ is much more expensive than the other two strategies.

Sensitivity to discounting. As our third example, we examine the impact of discount rate $v$ on the distribution of NPV. In Fig. 6, we demonstrate the distribution of $S$ computed for $v \geq 1$, and in Fig. 7 it is computed for $v<1$. We give two different graphs because we need two different horizontal scales. Both figures show that by increasing $v$ we increase the difference between the distributions, because, by increasing $v$, we increase the values $S$ can take, which leads to the shift of the probability mass to higher values.

Another observation is that whereas the distribution of $S$ in Fig. 6 is quite smooth, in Fig. 7 it is quite irregular. This can be explained by the following argument. When $v<1$, due to the presence of the powers of $v$ in Eq. (7), $S$ effectively becomes a sum of a small number of random variables. For 


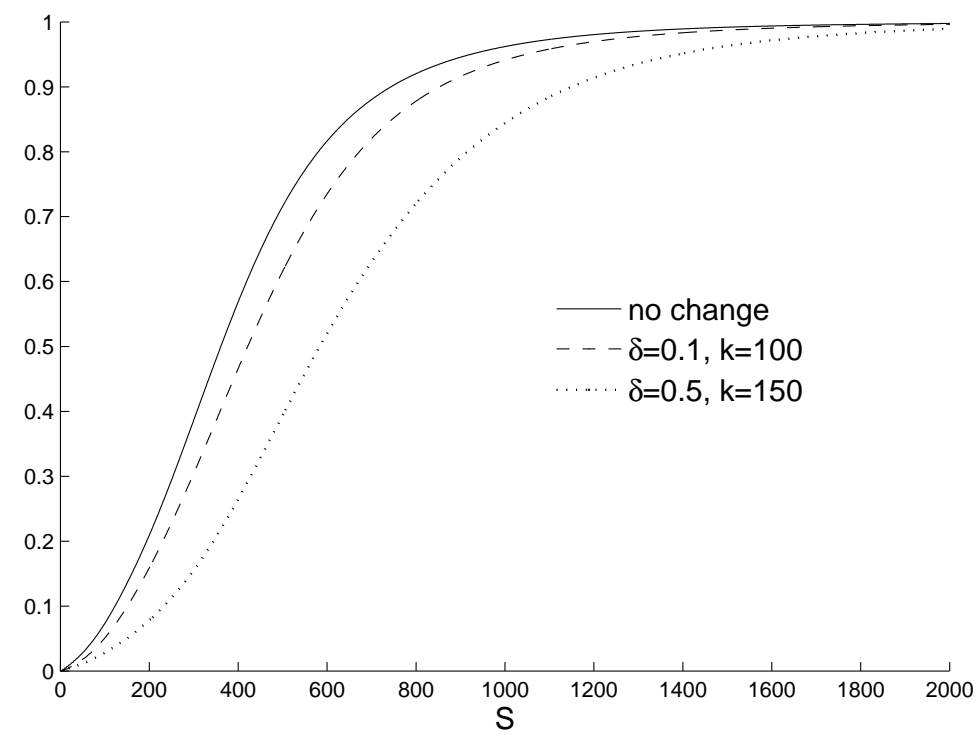

Figure 5: Markov reward model: distribution of $S$ for different treatment strategies with $\operatorname{cost} c=5$ $v=1.02, W_{i}, i \in\{A\}$ from Fig. 2, SW1911M

example, if $v=0.7$ already at time $t=10$ with maximal value of $X_{t}$ being $M=10$, the actual cost value does not exceed $v^{t-1} M=0.4$. Furthermore, in the Markov reward model the $X_{t} \mathrm{~s}$ are dependent random variables and may take the same values for successive values of $t$ due to the step structure of costs for health states in our example (see Fig. (2)). This explains the irregularity that we observe for $S$ for $v<1$. When $v=1.02, S$ is a sum of a large number of random variables and, despite their dependence, we can observe a smooth curve.

\section{$6 \quad$ Perspectives}

As our main contribution we indicate the development of two discrete time mathematical models to determine recursive equations for the distribution of NPV of a health dependent expense over a random phase-type time horizon. The lifetime and health of the individual are described by the $\mathrm{PH}$-aging model with the generator $Q$ (see (1)) of any structure.

Specifically, we have elaborated models with health dependent costs with constant and random costs for a given state and a model with iid costs. We have obtained recursive equations for the distribution of NPV by extending 


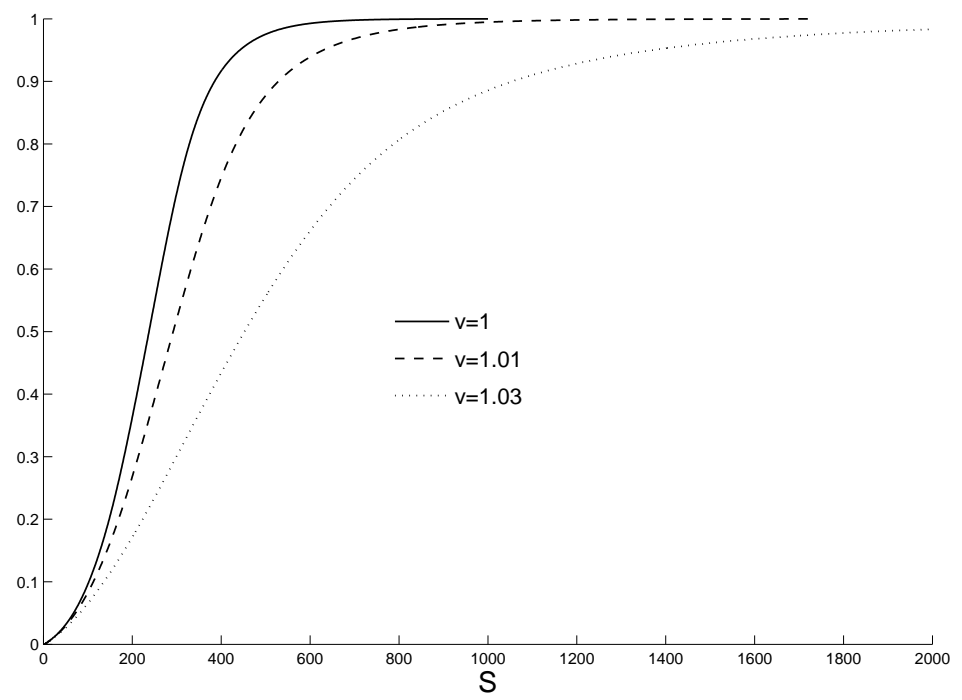

Figure 6: Markov reward model: distribution of $S$ for different $v>1$ $W_{i}, i \in\{A\}$ from Fig. 2, SW1911M

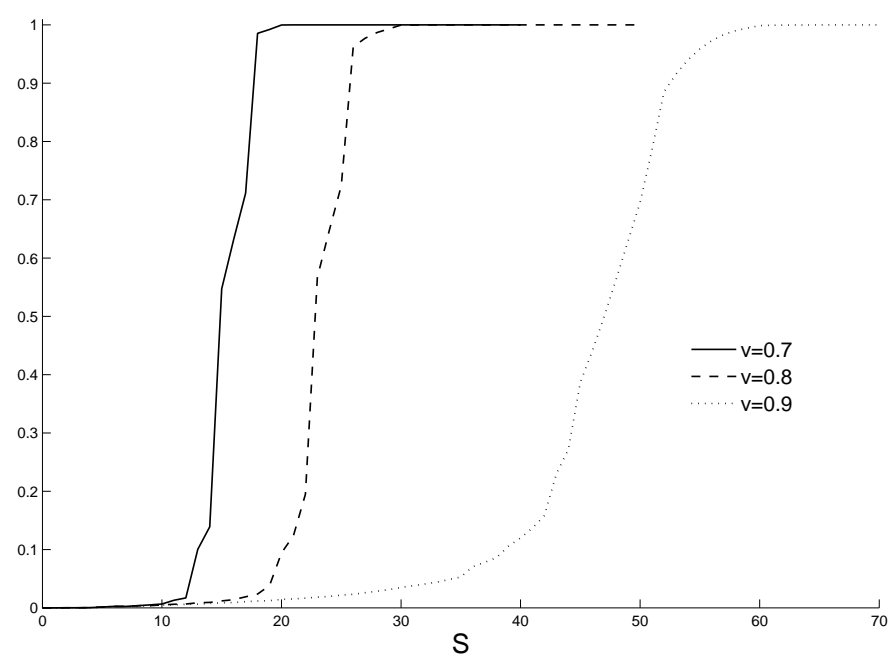

Figure 7: Markov reward model: distribution of $S$ for different $v \leq 1$ $W_{i}, i \in\{A\}$ from Fig. 2, SW1911M 
the procedure introduced in Panjer [1981]. For each of the models, we have also derived closed form expressions for the expectation of NPV.

Furthermore, we have proposed a parametrization procedure and a technique to estimate the financial impact of two health related events. The first one is related to longer survival in bad health states. In the second one, we estimate the NPV and future life expectancy for different treatment strategies, which we believe helps a decision process in health economics. We remark that the constructed scenarios can be adapted to particular needs and purposes, and can be used to describe more complex situations in health insurance and health economics. We have also investigated sensitivity of the NPV distribution to the discount rate.

We believe that the developed models and equations are general enough and may be applied to any other quantities where total discounted payments are correlated with the lifetime of an individual.

One natural extension of these results that we plan to examine in details is the continuous time version of the proposed models. Clearly, continuous time models would allow one for a more precise computation of the distribution of NPV with flexible time horizons. Our main mathematical approach uses fluid queue techniques, and it has been described in Govorun [2013].

Also, in our models we are interested in taking into account market fluctuations by having a more complex time dependent model for the discount rate $v$.

\section{References}

C. Castelli, C. Comberscure, Y. Foucher, and J.-P. Daures. Cost-effectiveness analysis in colorectal cancer using a semi-markov model. Statistics in medicine, 26:5557-5571, 2007.

M. F. Drummond, M. J. Sculpher, G. W. Torrance, B. J. O'Brien, and G. L. Stoddart. Methods for the Economic Evaluation of Health Care Programmes. Oxford university press, 2005.

K.-T. Eisele. Recursions for compound phase distributions. Insurance: Mathematics and Economics, 38:149-156, 2006.

J. C. Gardiner, Z. Luo, C. J. Bradley, C. M. Sirbu, and C. W. Given. A dynamic model for estimating changes in health status and costs. Statistics in medicine, 25:3648-3667, 2006.

M. Govorun and G. Latouche. Modeling the effect of health: phase-type approach. European Actuarial Journal, 4(1):197-218, 2014. 
M. Govorun, G. Latouche, and M.-A. Remiche. Profit test model for pension funds using matrix-analytic modeling. Proceedings of the Actuarial and Financial Mathematics Conference - Interplay Between Finance and Insurance, pages 87-94, 2011.

M. Govorun, B. L. Jones, X. Liu, and D. A. Stanford. Physiological age, health costs and their interrelation. submitted for publication, 2014.

Maria Govorun. Pension and Health Insurance: Phase-Type Modeling. $\mathrm{PhD}$ thesis, Université Libre de Bruxelles, http://theses.ulb.ac.be/ETDdb/collection/available/ULBetd-08262013-084245/, 2013.

Quentin Guibert and Frédéric Planchet. Construction de lois d'expérience en présence d'événements concurrents. Application à l'estimation des lois d'incidence d'un contrat dépendance. to appear in French Actuarial Bulletin, 2014.

G. Latouche and V. Ramaswami. Introduction to Matrix Analytic Methods in Stochastic Modeling. ASA-SIAM, 1999.

X. S. Lin and X. Liu. Markov aging process and Phase-Type Law of mortality. North American Actuarial Journal, 11:92-109, 2007.

H. H. Panjer. Recursive evaluation of a family of compound distributions. Astin Bulletin, 12:22-26, 1981.

S. Su and M. Sherris. Heterogeneity of australian population mortality and implications for a viable life annuity market. Insurance: Mathematics and Economics, 51:322-332, 2012.

X. Zhao and X. Zhou. Estimation of medical costs by copula models with dynamic change of health status. Insurance: Mathematics and Economics, 2012. 